\title{
Philosophiques
}

\section{Gestalt, subjectivité, négativité : l'interprétation heideggerienne du Surhomme et sa critique de la morphologie des cultures chez Spengler}

\section{Golfo Maggini}

Volume 26, numéro 1, printemps 1999

URI : https://id.erudit.org/iderudit/004954ar

DOI : https://doi.org/10.7202/004954ar

Aller au sommaire du numéro

Éditeur(s)

Société de philosophie du Québec

ISSN

0316-2923 (imprimé)

1492-1391 (numérique)

Découvrir la revue

Citer cet article

Maggini, G. (1999). Gestalt, subjectivité, négativité : l'interprétation heideggerienne du Surhomme et sa critique de la morphologie des cultures chez Spengler. Philosophiques, 26(1), 53-70. https://doi.org/10.7202/004954ar
Résumé de l'article

Notre article traite de l'interprétation heideggerienne du Surhomme ( Ubermensch ) chez Nietzsche en prenant en considération tout particulièrement sa désignation comme forme ( Gestalt ) dans les cours de 1939-1946 qui constituent, sous plusieurs aspects, la culmination du " différend " ( Auseinandersetzung ) avec lui. En effet, si anthropomorphie et subjectivité s'appartiennent mutuellement dans la métaphysique moderne, cette coappartenance trouve son achèvement chez le Surhomme. Elle indique par ailleurs la forclusion du sujet nietzschéen dans la logique de la négation, témoignage de la tentative heideggerienne pour « dialectiser » Nietzsche. C'est ainsi qu'une continuité s'établit entre sa position métaphysique et la dialectique hégélienne. Par ailleurs, nous nous efforçons de projeter cette interprétation réductrice sur la critique par Heidegger d'une des figures majeures du néohégélianisme au tournant du siècle, à savoir Oswald Spengler, telle qu'elle a lieu dans ses cours de l'herméneutique de la facticité (1919-1923). Notre stratégie interprétative est fidèle au principe interprétatif qui consiste à dire que le Heidegger d'avant Être et Temps, nous offre les outils nécessaires pour aborder certains motifs essentiels du développement ultérieur de sa pensée dont notamment sa confrontation avec la métaphysique.
Tous droits réservés @ Société de philosophie du Québec, 1999
Ce document est protégé par la loi sur le droit d'auteur. L’utilisation des services d’Érudit (y compris la reproduction) est assujettie à sa politique d'utilisation que vous pouvez consulter en ligne.

https://apropos.erudit.org/fr/usagers/politique-dutilisation/ 


\title{
Gestalt, subjectivité, négativité : l'interprétation heideggerienne du Surhomme et sa critique de la morphologie des cultures chez Spengler
}

\author{
GOLFO MAGGINI \\ SUNY \\ Stony Brook \\ U.S.A.
}

RÉSUMÉ. - Notre article traite de l'interprétation heideggerienne du Surhomme (Ubermensch) chez Nietzsche en prenant en considération tout particulièrement sa désignation comme forme (Gestalt) dans les cours de 19391946 qui constituent, sous plusieurs aspects, la culmination du « différend » (Auseinandersetzung) avec lui. En effet, si anthropomorphie et subjectivité s'appartiennent mutuellement dans la métaphysique moderne, cette coappartenance trouve son achèvement chez le Surhomme. Elle indique par ailleurs la forclusion du sujet nietzschéen dans la logique de la négation, témoignage de la tentative heideggerienne pour « dialectiser » Nietzsche. c'est ainsi qu'une continuité s'établit entre sa position métaphysique et la dialectique hégélienne. Par ailleurs, nous nous efforçons de projeter cette interprétation réductrice sur la critique par Heidegger d'une des figures majeures du néohégélianisme au tournant du siècle, à savoir Oswald Spengler, telle qu'elle a lieu dans ses cours de l'herméneutique de la facticité (1919-1923). Notre stratégie interprétative est fidèle au principe interprétatif qui consiste à dire que le Heidegger d'avant Être et Temps, nous offre les outils nécessaires pour aborder certains motifs essentiels du développement ultérieur de sa pensée dont notamment sa confrontation avec la métaphysique.

\begin{abstract}
This paper focuses on Heidegger's interpretation of the Ubermensch with a special emphasis on his designation as a form (Gestalt) developped mainly in the 1939-1946 lecture courses on Nietzsche. The belongingtogether of « unconditional » subjectivity and anthropomorphism reaches its completion in the «form » of the Ubermensch, while it also witnesses its forclosure in the logic of negativity. Consequently, a direct continuity is established between Nietzsche's metaphysical position and Hegel's dialectics. By taking this unexpected affinity as a starting point, we go back to Heidegger's early critique of hegelianism and especially of Spengler's project of a morphology of the cultures in his hermeneutics of facticity (1919-1923). This interpretative strategy unfolds according to the principle that the first steps on Heidegger's path of thinking offer us the necessary conceptual tools in order to come to terms with the later developments of his confrontation with metaphysics.
\end{abstract}

La fracture produite dans l'histoire de la subjectivité moderne par l'irruption du Surhomme est affirmée à plusieurs reprises dans le propos heideggerien. À l'égoïté du sujet chez Descartes, à l'individualité de l'esprit chez Hegel et aux courants philosophiques du XIX ${ }^{\mathrm{e}}$ siècle qui se situent à sa suite succède alors 
une "nouvelle empreinte de l'humanité " (eine neue Prägung des Menschentums $)^{\prime}$. À la différence du sujet égoïque et du sujet absolu qui ne sont pas encore " assez subjectifs », chez le Surhomme, la métaphysique moderne parvient à la détermination extrême et accomplie de son essence ${ }^{2}$.

"Type » (Typus) et " forme » (Gestalt) sont les termes qui s'alternent dans cette désignation. Heidegger cite à plusieurs reprises le fragment 713 de la Volonté de Puissance où Nietzsche écrit à ce propos : "il s'agit du type : l'humanité n'est qu'un simple matériel d'expérimentation, l'énorme excédent des mal-venus : un champ de ruines ${ }^{3}$ ». Chez le Surhomme, il ne s'agit pas d'une mutation quantitative qui amène à l'éventuel achèvement (Vollendung) de l'humanisme moderne mais d'un "saut » qualitatif. Il y va en effet de l'homme nihilistiquement inversé et par là même érigé en Gestalt.

C'est à un moment critique du cours de 1940 sur "Le Nihilisme Européen " que Heidegger donne une définition complète des concepts de forme et de type. En réunissant en lui la singularité de l'individu et l'homogénéité du sujet collectif, le type parvient à " une claire universalité de la même empreinte » (in einer klaren Durchgängigkeit derselben Prägung) inaugurant une hiérarchie nouvelle :

Dans la pensée nietzschéenne du surhomme, ce n'est pas un type d'homme particulier mais, pour la première fois, l'être-humain qui se voit pré-conçu sous la figure essentielle du type (in der Wesensgestalt des "Typus" vorausgedacht) ${ }^{4}$

Afin d'éclairer la désignation du Surhomme en termes d'empreinte (Prägung), il nous faut de toute évidence commencer par mesurer son envergure, puisque Heidegger lie ce terme non pas uniquement à la philosophie nietzschéenne mais aussi à toute forme de pensée métaphysique. Ainsi, dans le cours de 1941 sur "La métaphysique en tant qu'histoire de l'Être », la temporalité propre à toute configuration de présence est qualifiée d'empreinte :

Au commencement de son histoire, l'Être s'éclaircit en tant

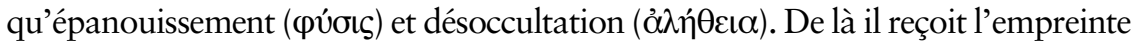
(Gepräge) de la présence et de la consistance au sens du demeurer[... $]^{5}$

I. Parmenides (1942/43), GA 54, Francfort, Klostermann, 1982, p. 204.

2. Nietzsche, vol. II, Paris, Gallimard, 1971, p. 54 (désormais sous le sigle NII).

3. Ibid., p. 246.

4. NII, p. 118. Nous tenons à signaler d'emblée une affinité qui suscite certes de nombreuses hypothèses. La même nécessité historiale qui fait du Surhomme chez Nietzsche une Gestalt qualifie aussi selon Heidegger l'Ange rilkéen dans la huitième des Élégies de Duino. Lors de l'achèvement de la métaphysique moderne, l'Ange rilkéen s'avère " être métaphysiquement le Même que la figure nietzschéenne de Zarathoustra ", ce qui renvoie ici, comme dans le cas du Surhomme, à la nécessité d' " une explication plus originaire de l'essence de la subjectivité " ( "Pourquoi des poètes ? ", 1946, Chemins qui ne mènent nulle part, Paris, Gallimard, 1962, p. 375-6; désormais sous le sigle Chemins). Dans le cas de Rilke, comme dans celui de Nietzsche, il s'agit de manifestations de la métaphysique parvenue à son achèvement soit dans les termes de la pensée, soit dans ceux de la poésie.

5. NII, p. 324. 
Or, ce même réseau conceptuel de l'empreinte et de l'empreindre désigne également l'expérience de l'Être propre à la métaphysique. Dans le fragment 617 de La Volonté de Puissance, repris et interprété par Heidegger à plusieurs reprises, Nietzsche écrit : " Imprimer au devenir le caractère de l'Être, voilà la suprême volonté de puissance ${ }^{6}$. » Nous nous efforcerons par la suite de comprendre l'usage du motif de la Gestalt dans ses multiples ramifications - forme, type, frappe, empreinte, figure, image - à la lumière de la confrontation heideggerienne avec la métaphysique en retraçant les origines de ce parcours au tout début de son enseignement philosophique et plus précisément aux cours et aux écrits qui marquent son élaboration d'une herméneutique de la facticité (1919-1923).

Cette remarque nous invite à revenir au sens dont est doté ici le terme Gestalt: "C'est de la simplicité propre à la volonté de puissance que découlent l'univocité, la fermeté, le poli de tous ses façonnements et de toutes ses empreintes (Prägungen), de toutes ses frappes (Schläge). c'est d'elle que résulte, c'est à elle que correspond ce qui est frappé d'une empreinte, le typique ". Vu sous cet angle, le Surhomme n'est pas qualifié de simple empreinte mais d' "empreinte (Schlag) d'une humanité laquelle pour la première fois se veut elle-même en tant qu'empreinte et s'en empreint elle-même (selbst zu diesem Schlag sich schlägt) ${ }^{7}$ ".

Cette définition repose sur la Volonté de puissance envisagée dans l' « inconditionnalité » (Unbedingtheit) de son essence. Le Surhomme n'est pas qualifié simplement de forme mais de forme essentielle (Wesensprägung/ Wesensgestalt), à travers laquelle se réalise la fixation d'essence de celui qui était jusqu'alors le dernier homme (letzte Mensch). Ce qui détermine l'anthropomorphie dans la métaphysique de la subjectivité parvenue à sa fin, c'est son pouvoir de formation (Gestaltung) et de façonnement (Prägung) de l'étant. En réalité, afin d'éclairer les fondements métaphysiques de la Gestalt, il nous faudrait mobiliser un réseau complexe de renvois dont les points d'ancrage sont à chercher auprès de l'ỉó $\alpha$ platonicienne - provenance historiale qui fera l'objet notamment de la lettre-réponse de 1955 à Ernst Jünger $^{8}$-, de la cogitatio cartésienne mais aussi et surtout des motifs de système absolu et de négativité dans la métaphysique de l'idéalisme allemand, notamment chez Hegel. Par la suite, nous aborderons l'étape décisive qui constitue l'antécédent historial pour le surgissement du Surhumain, à savoir la subjectivité « inconditionnée » (unbedingte) dans l'idéalisme allemand, avant de parvenir au cœur de notre questionnement, à savoir la négation comme

6. Ibid, p. 231.

7. Ibid, p. 248, 250 (souligné dans le texte).

8. Dans Contribution à la question de l'Être (1955), la désignation du Surhomme

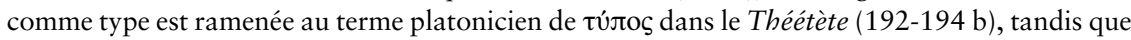
la Gestalt est qualifiée de retrait absolu de toute transcendance dans la rescendance (Rückstieg, Reszendenz). La rescendance par la forme se produit de telle façon que cette dernière "se représente " en se présentant ainsi une fois de plus dans ce qu'elle a empreint de son caractère. L'entreprise de formation (Gestaltung) de l'étant qui obéit non pas à un lien causal mais au rapport du sceau à l'empreinte correspond de façon nécessaire à la désignation de l'Être de l'étant comme production de la présence sous la figure de la Volonté de puissance. La reduplication de l'essence qui lui est propre témoigne de l'essence anthropomorphique de la métaphysique moderne (Questions I, Paris, Gallimard, 1968, p. 215). 
essence de la «forme » et son renvoi à la critique heideggerienne du projet d'une morphologie (morphè/Gestalt) des cultures dans les années vingt.

\section{De la subjectivité « inconditionnée » dans la métaphysique de l'idéalisme allemand : «Gestalt » et anthropomorphie}

Si, d'une part, Heidegger affirme que « dans l'avènement des Anthropologies, Descartes fête son suprême triomphe ${ }^{9}$ ", il pose, d'autre part, que la subjectivité du Surhomme correspond à une aggravation (Aufblähung) de l'anthropologie moderne ${ }^{10}$, due essentiellement au passage de la subjectivité égoïque, essentiellement « conditionnée » (bedingte), à la subjectivité conçue dans l'inconditionnalité de son essence :

Il nous faut [...] attirer l'attention sur le fondement d'essence de la fatalité historiale de l'histoire de la métaphysique - en tant qu'une histoire de la vérité de l'Être. Cette indication nous permet du même coup d'éclaircir une distinction que nous avons déjà pratiquée plus d'une fois : la distinction entre la subjectivité conditionnée et celle inconditionnée (die Unterscheidung der bedingten und der unbedingten Subjektivität $)^{11}$

Le pas décisif dans cette évolution devient possible avec la transition de la métaphysique de la représentation à la métaphysique de la volonté dont Leibniz est la figure inaugurale. La philosophie hégélienne de l'Esprit, mais aussi l'idéalisme allemand dans son ensemble, sont marqués par la prééminence de la volonté, notamment de la "volonté de système " (Wille zum System). Chez Hegel, notamment dans La Phénoménologie de l'Esprit qui sert ici de texte de référence, la raison fait de la subjectivité inconditionnée un volontaire se savoir soi-même.

1 - Dans cette étape de l'achèvement (Vollendung) de la métaphysique moderne, la volonté est "volonté de l'esprit ", "volonté pensante ", de sorte que, par phénoménologie chez Hegel, on entend «la manière dont l'inconditionnée subjectivité, en tant que l'inconditionné acte de représenter apparaissant à soi-même (penser) est elle-même l'être de tout étant ${ }^{12}$ ". Ce qui est d'importance majeure pour le propos tenu ici, c'est précisément le lien intime établi entre la Phénoménologie et la Logique hégéliennes. La place de la Logique dans la Phénoménologie est justifiée par le fait que c'est en elle seulement que l'apparaître à soi-même du sujet amène celui-ci à l'inconditionnalité de son essence. À l'encontre de l'écart établi entre la Phénoménologie et le système de la Logique dans le cours de 1930/31 sur la Phénoménologie de l'Esprit, le renvoi de la dialectique hégélienne à la métaphysique de la subjectivité procède

9. "L'époque des conceptions du monde " (1938), Chemins, p. 130.

Io. Die Metaphysik des deutschen Idealismus (1941), GA 49, Francfort, Klostermann, 1991, p. 71.

I I. NII, p. 154.

I 2. Ibid., p. 240. 
désormais de la coappartenance de la Phénoménologie et de la Logique. Le tournant vers l'inconditionnalité de l'essence subjective s'annonce en effet sous la figure de l'absolu. Celui-ci s'identifie à la subjectivité parvenue à son achèvement dans l'élément du savoir (Wissen). Raison (Vernunft) et absolu sont d'autres noms pour l'essence métaphysique de la subjectivité.

Quant à l'Esprit hégélien, il doit être conçu comme esprit absolu qui rassemble et réunit en soi par une médiation ${ }^{13}$. Il s'identifie ainsi à la certitude de la représentation comme représentation inconditionnelle de soi-même, de sorte que l'absolu n'est pas seulement en soi ( $\pi \rho \tilde{\omega} \tau o v \varkappa \alpha \theta^{\prime} \alpha v i$ 'ó), mais aussi pour nous $(\pi \rho o ̀ \varsigma \dot{\eta} \mu \tilde{\alpha} \varsigma)^{14}$. Par conséquent, le premier achèvement de la métaphysique moderne dans l'absoluité de l'Esprit s'effectue, du côté de l'objet, par la réduction de la réalité du réel à l'effectivité et, du côté du sujet, par la subjectivité entendue en termes de conscience de soi et par là même identifiée à la Science (Wissenschaft). C'est dans la disposition du se-savoir soi-même qu'advient l'essence du sujet comme certitude absolue de soi, de sorte que celuici ne soit "disposé " qu'à la Science : "La philosophie est la Science, parce qu'elle veut la volonté de l'absolu, c'est-à-dire celui-ci en son absoluité15. »

Ce sont ces déterminations essentielles qui conditionnent la mutation de la métaphysique moderne en anthropomorphie, puisqu'en cette dernière, "d'une manière générale l'étant en tant que tel s'interprète selon les modalités humaines de l'être, indépendamment du fait que l'interprétation s'effectue par l'homme $^{16}$ ». Ce virage fait l'objet d'examen à deux endroits qui font partie des cours des années quarante sur Nietzsche. Le premier est le développement réservé à ce sujet dans le cours de 1940 sur "Le Nihilisme Européen ", qui porte le titre "Métaphysique et anthropomorphie ». Le second est l'analyse détaillée de l'anthropomorphie dans le cours de 1941 sur la métaphysique de l'idéalisme allemand. Schelling et Nietzsche sont les deux figures principales de l'anthropomorphie moderne. La différence entre les deux repose en effet sur la conception divergente du statut de la volonté qui constitue le noyau de leur position métaphysique fondamentale. En tout état de cause, l'anthropomorphie témoigne de l'élément déterminant de la métaphysique moderne amenée à sa fin, voire à l'inconditionnalité de son essence. Or, dans la mesure où « la métaphysique est anthropomorphie - le fait de structurer et de concevoir le monde à l'image de l'homme'7 ", le Surhomme n'est pas tout simplement la mesure de l'étant, comme c'était le cas pour l'è $\gamma \hat{c}$ chez Protagoras. Il constitue au contraire la morphé selon laquelle se façonne toute chose.

Dans le cours du semestre d'été 1941 sur l'idéalisme allemand, la tripartition scolastique de l'étantité de l'étant constitue le point de départ de l'interrogation : le plus étant parmi les étants (

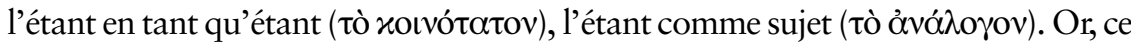
troisième élément rend explicite l'essence de l'homme comme Ebenbild Gottes :

I3. Cf. Schelling, Appendice (1941), Paris, Gallimard, 1977, p. 322.

I4. GA 49, p. 119.

I 5. "Hegel et son concept de l'expérience " (1942/1943), Chemins, p. 164-165.

I6. NII, p. 105.

I7. Ibid., p. 104. 
À l' « analogisation » propre à l'interprétation de l'étant dans sa totalité et dans sa diversité vient encore se joindre, depuis que l'ens est interprété comme certum et comme subjectum [...] l'antithétique de la conscience et de la conscience-desoi, antithétique qui conduit logiquement (sous la contrainte de la vérité de

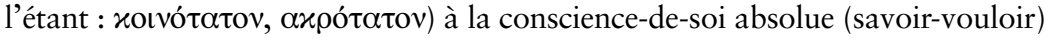
et qui exige par conséquent la dialectique ${ }^{18}$

La doctrine scolastique de l'analogie de l'étant (die Lehre von der " Analogie des Seienden ») est ainsi à l'origine de l'anthropomorphie moderne. Cette dernière est qualifiée par le passage de la conception de l'homme comme centre de l'étant - ce qui correspond aux moments cartésien et leibnizien - à celui devenu sa figure unique. Le monde et son fondement, Dieu, ne sont que des figures de l'homme conçu dans l'inconditionnalité de son essence : tout étant emprunte une

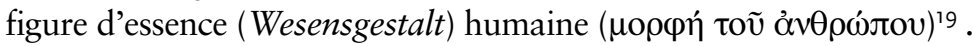

L'insistance avec laquelle Heidegger affirme que l'anthropomorphie vient à la suite du théomorphisme s'appuie sur la continuité historiale entre la philosophie scolastique et la pensée moderne. Elle constitue par conséquent un trait essentiel de la métaphysique moderne de la subjectivité :

[...] dans la mesure où l'Être devient actualitas (réalité), l'étant est le réel qui se détermine par l'agir au sens de faire qui cause et provoque. La réalité agissante du faire humain et du créer divin se déterminent ainsi de la même façon ${ }^{20}$.

C'est en raison de cette affinité historiale que, lors de l'achèvement de la métaphysique moderne, toute interprétation de l'étant est essentiellement anthropomorphique. De là provient aussi sans doute la fonction particulière de cette dernière chez Heidegger, puisqu'elle n'indique pas une question régionale comme c'est le cas chez Leibniz et Malebranche. L'anthropomorphie apparaît au contraire dans son rapport essentiel à l'anthropologie dont elle est la figure finale.

Si chez Descartes Dieu est encore la lumière qui éclaire la connaissance humaine, dans la métaphysique de la subjectivité inconditionnée, c'est l'homme qui s'érige en Deus Faber. Or, construction de l'étant, anthropomorphie et système sont censés s'entrappartenir, dans la mesure où absoluité et anthropomorphie découlent de l'essence volontative de la subjectivité. Par ailleurs, la construction de la totalité de l'étant s'inscrit d'avance dans le cercle du réel considéré dans sa provenance historiale de l'exigentia essentiae leibnizienne : "Le cum- entendu comme unité au sens de l' "unité" “de" l'être (l'unité de l'entrée-en-présence, telle qu'elle appartient à l'être lui-même). (Unité de la proponibilité, de la représentéité de la représentation se re-présentant à soi-même ${ }^{21}$ ) » Or, la réalité du réel chez Hegel va de pair avec l'inconditionnalité de la subjectivité envisagée dans sa coappartenance avec le système absolu. Il s'agit

I8. Schelling, Appendice (1941), p. 321.

19. Nietzsches Metaphysik Einleitung in die Philosophie : Denken und Dichten (1941), GA 50, Francfort, Klostermann, 1990, p. 110.

20. NII, p. 333

21. Schelling, p. 292. 
par là d'affirmer l'identification de l'unité de la représentation représentant ellemême à la subjectivité du sujet. La conséquence directe en est le lien intrinsèque de la métaphysique du système avec l'anthropomorphie. Du côté du sujet, le système est l'essentification de la volonté absolue elle-même, tandis que, du côté de l'objet, il est le mode de réalisation du réel, le mode d'exister de l'existant ${ }^{22}$. C'est à partir de cette réflexion intense et originale sur l'idéalisme allemand, sur Hegel et Schelling en particulier, qu'il devient possible de considérer la figure ultime de la doctrine de l'analogie de l'étant dans la métaphysique : Nietzsche. Ainsi, à la lumière du rapport historial de l'anthropomorphie au système, nous sommes en position d'évaluer le poids de l'affirmation heideggerienne selon laquelle Nietzsche parachève l'anthropomorphie moderne car il demeure à son insu un métaphysicien du système : ni il n'a conçu ni il n'a essayé de concevoir son essence (er das Wesen nicht begriff und begreifen konnte) ${ }^{23}$. Les fragments les plus fréquemment cités et commentés par Heidegger pour explorer les assises métaphysiques de la Gestalt témoignent du lien essentiel établi entre le processus d'humanisation de l'étant et la subjectivité explicitée en termes de maitrise. Nietzsche écrit : " humaniser le monde, c'est-à-dire nous sentir en lui de plus en plus en tant que maîtres ${ }^{24}$. Cette affirmation renvoie expressément à la désignation du Surhomme comme "forme " : celui-ci ne fait en effet que consolider l'anthropomorphie inhérente à la métaphysique.

Revenons brièvement à ce que Heidegger désigne comme premier trait du système dans la métaphysique de l'idéalisme allemand, à savoir sa capacité de se produire soi-même et par là même de produire l'étant dans sa totalité. Pour la métaphysique parvenue à son achèvement, le vrai est le système, ce qui s'accomplit à travers le déploiement de soi-même. Dans son commentaire de l'Introduction à la Phénoménologie de l'Esprit (1941/42), le système est défini comme ce qui rassemble dans une conscience unique qui est conscience inconditionnée du soi et qui se constitue dans ce rassemblement pour soi. Les formes de la "systématique " (Systematik) ne sont en soi que "des figures d'essence cachées de la conscience, son objectivité pour elle-même (le nouveau vrai objet $\left.{ }^{25}\right) »$.

De même que dans le cas de l'ordo et de l'analogia entis scolastiques, Heidegger affirme ici la coappartenance du système et de l'anthropomorphie dans la métaphysique de la subjectivité "inconditionnée ». Or ce rapport est alors formulé dans des termes tout à fait différents, puisque le système est censé dire l'essence de l'étant comme tel en totalité. L'être se conçoit ainsi en termes de sustasis. Dans la mesure où l'être et la subjectité font un, la "systématique " constitue l'être de l'étant ${ }^{26}$. Le Surhomme s'avère être

22. GA 49, p. 139 (notre traduction).

23. Beiträge zur Philosophie (Vom Ereignis), 1936-38, GA 65, Francfort, Klostermann, 1989 , p. 89.

24. V.P., ${ }^{\circ} 614$ (cité dans NII, p. 245-246).

25. Hegel - 1. Die Negativität 2. Erläuterung der Einleitung zu Hegels "Phänomenologie des Geistes» (1938-39; 1941-42), GA 68, Francfort, Klostermann, p. 137. 26. "Le système dit l'essence de l'étant comme tel en totalité, c'est-à-dire l'étantité de l'étant. Être en tant que systasis: le systématique constitue l'être de l'étant, parce que l'être est 
l'étape ultime dans ce processus anthropomorphique qui s'achève dans la métaphysique de la volonté27.

\section{Gestalt et système : la question de la négativité}

Nous avons tenté de retracer brièvement le parcours qui amène Heidegger à l'affirmation fondamentale que l'anthropomorphie est le trait essentiel de la métaphysique moderne parvenue à sa fin dans l'idéalisme allemand. L'accomplissement de cette fin se fonde sur la prépondérance du système, ce qui présuppose la reconnaissance du rapport étroit entre la métaphysique du système et le primat de la représentation. À partir de ces éclaircissements préliminaires, nous traiterons de la question de la négativité, qui constitue selon nous la pierre d'achoppement de l'interprétation heideggerienne du Surhomme. Ainsi lit-on dans le cours sur "La métaphysique de Nietzsche »:

Le «sur» dans le nom de "surhomme » contient une négation et signifie le fait d'aller par-delà et au-delà de l'homme tel qu'il a été jusqu'alors. Le "non » de cette négation est inconditionnel, absolu, dès lors qu'il procède du « oui » de la Volonté de puissance et qu'il vise directement l'interprétation platonicienne, chrétienne et morale dans toutes ses formes dérivées, manifestes ou cachées ${ }^{28}$

Le lien établi entre les motifs du système et de la négativité devient expresse notamment dans l'écrit majeur des années 1936-1938 intitulé Beiträge zur Philosophie. C'est ici que la question de la négativité (Negativität) entre en scène. Il s'agit en effet de trancher nettement entre celle-ci et l'intimité du néant originaire (die Innigkeit des Nichts) qui s'identifie à l'essence litigieuse dans l'Être (das Strittige im Sein). Cette divergence aura comme point de départ la critique adressée aux deux métaphysiciens du système et de la négativité : Hegel et Nietzsche ${ }^{29}$. L'entrelacement des deux motifs sera repris et approfondi par la suite dans le cours de 1938/39 sur le concept hégélien de négation. Or, ce qui est mis en avant ici, c'est précisément le rapport entre la subjectivité, la conscience absolue de soi et la négativité comme l' « énergie de la pensée (absolue) 30 ». Ce

maintenant subjectité. Penser cette subjectité, telle est la tâche essentielle de la métaphysique " (Schelling, p. 306).

27. Selon la remarque éclairante de Reiner Schürmann : "L'anthropomorphie est nécessaire pour l'achèvement de la métaphysique parce que quand l'homme ne rencontre plus que lui-même dans les étants, le "meta-", le renvoi à une réalité différente du réel, s'est abîmé dans la reduplication de la volonté " (Le principe d'anarchie. Heidegger et la question de l'agir, Paris, Seuil, 1982, p. 242).

28. NII, p. 234-235.

29. GA 65, p. 264. Cf. Chemins, p. 131, 117. De même, dans le cours de 1941 sur l'idéalisme allemand, les quatre définitions de l'acte de représenter - über sich hinaus streben, Unterscheiden/Negativität, "Werden ", zu sich selbst bringen/sich vor-stellen — renvoient à la représentation de l'unité, à la présentification du multiple dans le recueillement qui convient à la métaphysique de la volonté (GA 49, p. 101).

30. GA 68, p. 27. 
dernier thème constitue en effet la réplique à la question de l'Être proposée par la métaphysique au moment de l'achèvement de ses possibilités essentielles. Mais il témoigne aussi d'une déficience inhérente à celle-ci, à savoir le fait qu'au sein d'elle la négativité demeure non-interrogée (fraglos) ${ }^{31}$.

À plusieurs reprises, Heidegger affirme l'écart absolu entre, d'une part, toute forme de métaphysique opérant à partir de la négativité dont Hegel est la figure principale et, d'autre part, la pensée du fondement sans-fond ( $A b$ grund). Or, Nietzsche se situe à la suite des métaphysiciens de l'idéalisme allemand et de Hegel en particulier, ce qui nous amène à considérer qu'un abîme s'instaure entre le Surhomme capté dans la logique de la la négativité et le néantiser (Nichtigen) propre à la philosophie qui assume le passage à l'autre commencement de la pensée.

Nous voici donc parvenus au rapport établi entre les motifs métaphysiques du système et de la négativité à travers quelques réflexions sommaires qui se déploient dans le cours de 1936 consacré à Schelling. Il s'agit ici pour Heidegger de montrer d'emblée que l'inversion (Umkehrung) considérée comme la réalité historiale propre à la métaphysique nietzschéenne clôt la démarche d'achèvement entamée par l'idéalisme allemand. Après avoir cité trois passages où Nietzsche dénonce avec acharnement le système comme manifestation de la montée du nihilisme, Heidegger conclut que sa volonté de renoncer au système est nécessaire, et cela, non pas parce que le système serait en soi quelque chose de vain, mais au contraire parce qu'il est ce qu'il y a d'essentiel ${ }^{32}$. Il offre par la suite deux définitions possibles du système à partir du terme grec de sunsthmi : il s'agit soit d'un ajointement interne (Gefüge) prétendant à l'authenticité, soit d'un rassemblement inauthentique. On assiste sans doute ici à l'un des rares indices d'une évaluation positive du concept de système chez Heidegger. Ce double renvoi fait défaut par la suite : le système n'emprunte plus, même partiellement, un caractère ajointé (Fugencharakter), mais il est au contraire entièrement englouti dans la domination métaphysique de l'unité33. L'élément déterminant de cette bifurcation est l'absence de limites qualifiant le système dans la métaphysique achevée, détermination d'autant plus significative que la limite est ici un autre terme pour désigner l'abritement (Bergung), l'être-jeté (Geworfenheit) et finalement la jointure (Fuge). Contrairement à l'essence subjective de la "systématique " moderne qui aboutit à la « forme », la figure " ajointée », délimitée, préserve la possibilisation historiale du Dasein.

Le terme grec de limite ( $\pi \dot{\varepsilon} \rho \alpha \varsigma$ ) fera aussi l'objet du cours de 1942/43 sur Parménide. La limite n'indique pas ici un point d'arrêt mais l'élément à travers lequel quelque chose émerge, ce qui figure (gestaltet) l'étant en le délimitant de telle ou telle façon, afin que celui-ci puisse se fixer dans le constant advenir à la présence. Or, le "figurer ", le laisser-advenir ne correspond nullement à la saisie métaphysique de la Gestalt mais à ce qui se désigne dans ce même texte en termes d'entre-deux (Zwischen). Ainsi, le

3I. "Die Negativität ist sowohl im System der Vollendung der abendländischen Metaphysik fraglos, als auch überhaupt innerhalb der Geschichte der Metaphysik » (ibid, p. 39).

32. Schelling, p. 51.

33. GA 54, p. 137; cf. GA 65, p. 136-137. 
discours du praw fait apparaître l'expérience de désubjectivation propre à la structure " ajointée » (fügende) de l'entre-deux ${ }^{34}$.

C'est précisément en raison de la coappartenance du système et de la négativité que, dans le cours de 1936 sur Schelling de même que dans celui de 1941 sur l'idéalisme allemand, le système compris comme un rassemblement interne est censé porter en lui l'élément constitutif du mal (Böse) désigné en termes de désaccord (Zwietracht), de litige (Streit), de " dis-jonction " $(U n-f u g)$ : "Le mal est pensé parce qu'en ce discord le plus extrême, en cet authentique discord qui est dis-jonction, c'est du même coup l'unité de l'ajointement de l'étant en totalité qui doit nécessairement apparaître avec le plus de relief ${ }^{35}$.»

Cette approche se situe en effet dans le droit fil des réflexions déployées dans les Beiträge zur Philosophie. Dans cet ouvrage charnière, le système se situe définitivement sur le plan de la négativité dialectique en s'opposant ainsi à la structure de l'ajointement $(F u g)^{36}$. Par conséquent, les traits de la négativité et du système constitutifs de la Gestalt se placent désormais au cœur de la position historiale de Nietzsche. La négativité, désormais considérée comme l'élément constitutif du système, constitue le noyau de la lecture heideggerienne du Surhomme en faisant de ce dernier l'étape finale dans la métaphysique moderne de la subjectivité.

La question de la subjectivité envisagée dans l'horizon de l'histoire de l'Être qui viendra plus tard au centre de son interrogation n'est pas encore présente dans le cours de 1936 sur Schelling. La "volonté de système " (der Wille zum System) et le primat de l'absolu seront liés par la suite à la subjectivité du sujet moderne considéré dans l'inconditionnalité de son essence, puisque le système devient le nom pour l'étant comme tel dans sa totalité ${ }^{37}$. Or, la domination métaphysique de l'unité sous la figure du système n'est possible que dans la mesure où l'Être de l'étant est déterminé par la subjectivité. Avec la mutation de l'essence de la vérité en certitude et la préfiguration de l'Être en tant que re-présentéité du se-représenter, c'est l'essence de la subjectité qui se déploie, et de là découle aussi la nécessité historiale du système ${ }^{38}$. Par conséquent, le

34. GA 54, p. 163; Heraklit. 1. Der Anfang des abendländischen Denkens (Heraklit) 2. Logik. Heraklits Lehre vom Logos, GA 55, Francfort, Klostermann, 1979, p. 369. Sur la désignation du préfixe Fest- du verbe Feststellen comme " cerné d'un contour, enchâssé dans sa limite $(\pi \varepsilon ́ \rho \alpha \varsigma)$, amené dans le tracé du trait » et puis comme " figé dans l'immeuble de la sécurité », synonyme du «cum » du « constituer » : "L'origine de l'œuvre d'art ", Supplément, Chemins, p. 94.

35. Schelling, p. 306.

36. GA 65, p. 264-268.

37. « Le système : unité — oủoí $\alpha$-Ẽv en tant qu'unité du "tenir-ensemble" (consistere) <au sens de l'ensemble se tenant debout> devant la conscience, pour celle-ci. Le "tenir-ensemble" détermine l'essence de l'unité. Toutefois, il faut que l'unité soit elle-même déterminée et questionnée dans la question concernant la vérité de l'Être » (NII, p. 371).

38. Ibid., p. 370-371. 
système dans son absoluité, c'est-à-dire comme figure de la totalité imprégnée de négativité, renvoie à l'étantité de l'étant identifiée à son tour à la subjectivité du sujet. Dans l'idéalisme allemand, l'idée signifie l'apparition de soi-même-à-soimême de l'étant dans le savoir absolu 39 .

Il devient évident par là que la différence entre la connaissance transcendantale chez Kant et le système hégélien de la conscience de soi repose sur la spécificité de ce dernier, à savoir la prise de conscience de la signification de la négativité donnant accès à "l'origine d'essence de l'“ob-stant” (l'objet) en sens absolu ${ }^{40}$ ». Le transcendantal n'est pas encore l'inconditionné (unbedingte). L'Esprit, la subjectivité du sujet chez Hegel, s'avère être « plus absolu " (absoluterer) que le moi de l'aperception transcendantale chez Kant : le sujet transcendantal est encore, d'une certaine façon, essentiellement conditionné (bedingte). Or, en s'interrogeant sur ce qui constitue l'essence du système absolu, Heidegger conclut qu'il s'agit de la négativité en tant que distinction (différenciée) de la re-présentation qui se représente elle-même. Vue sous cette angle, la négativité appartient ensemble avec l'in-conditionnalité. Le " in- " privatif est utilisé ici au sens de " à l'écart » du conditionné, au sens de mettre à l'écart et de supprimer, de laisser derrière soi, et cependant de tenir, de conserver et de sur-élever (Auf-beben $)^{4}$.

À l'encontre du sujet transcendantal, la conscience hégélienne de soi est l'objet d'elle-même, d'où la nécessité de formuler le concept métaphysique d'unité en termes de système absolu. Le virage critique se produit avec la nouvelle lecture de l'idéalisme allemand qui débute notamment avec l'élaboration des Beiträge zur Philosophie (1936-38). Comme il sera affirmé par la suite dans les cours sur Nietzsche, la philosophie de la volonté en tant que figure finale de la modernité est essentiellement une métaphysique du système : " dès que l'être a acquis l'essence de la volonté, il est en soi-même systématique et (par soi-même) un système ${ }^{42}$ ».

Dans les Beiträge, nous assistons au développement des conditions métaphysiques qui autorisent ce renvoi, même si la question de la subjectivité n'est pas placée ici au premier plan de façon explicite. La métaphysique du système trouve son accomplissement chez Nietzsche, notamment dans sa conception du Surhomme comme Gestalt envisagée dans la stricte continuité du système absolu hégélien. En effet, ce développement constitue la première étape dans un parcours où l'affinité de Nietzsche avec Hegel est maintes fois affirmée sur deux plans importants : premièrement, la question du système et, deuxièmement, la considération de la "métaphysique de la vie » (die

39. La critique du concept de totalité, concept constitutif selon Heidegger de la métaphysique, s'achèvera dans l'essai sur "La constitution onto-théologique de la métaphysique » (1957). Il s'agira du «avec » de l'énoncé « tout A est A »explicité comme " tout A est lui-même le même avec lui-même ": « de là vient que, d'un bout à l'autre de l'histoire de la pensée occidentale, l'identité se présente avec le caractère de l'unité » (Questions I, p.258-259) cf. Schelling, p. 165.

40. NII, p. 373.

4I. Schelling, p. 317.

42. Ibid., p. 364. 
Metaphysik des Lebens) chez Nietzsche dans la continuité de la réalité du réel effectif (die Wirklichkeit des Wirklichen) chez Hegel. Dans les deux cas, la logique de l'inversion est l'élément qui assure la continuité du schéma historial :

Le Surhomme nie sans doute l'essence jusqu'alors prévalente de l'homme, mais la nie de façon nihiliste. Sa négation vise ce qui jusqu'alors distinguait l'homme, la raison. L'essence métaphysique de celle-ci consiste en ce que, au fil conducteur de la représentation, l'étant dans sa totalité se projette et s'interprète en tant que tel ${ }^{43}$.

Ainsi, chez Nietzsche, l'inversion qui aboutit à la négation du système, au "non-systématique » (das Un-systematische), n'est que l'ultime confirmation de la métaphysique du système. Dans le cours de 1941 sur «Projets pour l'histoire de l'Être en tant que métaphysique ", Heidegger détermine les deux traits essentiels de la métaphysique moderne. Il s'agit, en premier lieu, de la prédominance du système absolu dans l'idéalisme allemand et, en second lieu, de sa non-essence en tant qu'achèvement extrême de la métaphysique. Or, l'affirmation que la volonté de système n'est qu'un "manque de sincérité » demeure inefficace dans la mesure où Nietzsche n'arrive pas à saisir le système comme l'élément fondateur de la modernité.

La décision (Entscheidung) qui fraie le chemin vers l'autre commencement (anderer Anfang) de la philosophie s'érige donc contre l'opposition avortée à la métaphysique du système chez Nietzsche. L'écart absolu entre le concept métaphysique du système et des concepts tels que la décision ou l'entre-deux (Zwischen) entre le commencement initial de la philosophie et son « autre commencement » renvoie finalement à la différence essentielle entre système et ajointement $(F u g)^{44}$. En fait, dans cette période marquée par un tournant critique dans l'interrogation heideggerienne sur le sort de la métaphysique, la tâche fondamentale de la pensée préparatoire à l' "autre commencement » est déterminée par la mise en question de la catégorie de l'unité subordonnée au primat de la présence ${ }^{45}$.

Au début du cours de 1942 qui porte sur la question de la négativité chez Hegel, il est question de l'essence inquestionnée de la négativité et du rapport de l'homme à l'Être comme étant les questions authentiques de l'anthropomorphie ${ }^{46}$. Cette affirmation va dans le sens de la conviction fondamentale que l'opposition entre l'essence systémique et la structure ajointée (fügende) du Dasein est l'axe du " différend » avec la métaphysique nietzschéenne, au moins pendant la période que nous interrogeons à présent : le négatif " est au fond le même que la conscience de soi absolue ${ }^{47}$ ".

Quant à Nietzsche, il s'agit pour Heidegger d'affirmer à nouveau que sa conception du devenir dépend de la négativité hégélienne. Nietzsche fait en

43. NII, p. 235.

44. GA 65, p. 89, 460, 458, 473; GA 45, p. 145.

45. Ibid, p. 460.

46. GA 68, p. 15.

47. Ibid., p. 27. 
effet un usage « restreint » du concept métaphysique d'Être au même titre que Hegel. C'est par là même que l'affinité entre les deux penseurs sera mise au clair à la lumière de leur fondement historial (geschichtlich) commun. Ainsi, dans son explication de l'énoncé nietzschéen sur l'Être comme " la dernière vapeur de la réalité évanouissante ", Heidegger le rapproche de la négation hégélienne. Le devenir chez Nietzsche ne sera désormais entendu que comme une médiation : "Hegel : le Néant comme la simple indéterminabilité et l'absence de médiation - l'absence de pensée comme telle. Nietzsche : l' "Être”, la dernière vapeur d'une réalité évanouissante ${ }^{48}$ ".

\section{Les origines de la critique heideggerienne de la Gestalt : Spengler et le projet d'une morphologie des cultures}

Loin de se détacher du parcours philosophique dans lequel s'engage d'emblée Heidegger, sa qualification du Surhomme comme Gestalt se situe au contraire à la suite d'une polémique féroce contre l'historicisme et le relativisme entamée très tôt dans son itinéraire philosophique. La critique des concepts de type et de typologie apparaît en effet pendant son enseignement à Fribourg (19191923). Elle s'inscrit dans le cadre de sa critique virulente des courants posthégéliens de la philosophie de l'histoire dont la philosophie de la culture (Kulturphilosophie) chez Spengler constitue une des figures principales. Sa typologie des cultures, de même que la psychologie jasperienne des « conceptions du monde » (Weltanschauungen), sont des manifestations du post-hégélianisme captives de la prétention à une histoire universelle ${ }^{49}$. Par là, il s'agit pour Heidegger de se battre contre le principe néo-kantien de la validité universelle (Allgemeingültigkeit). Ainsi, une relation étroite se tisse entre sa critique de la pensée «typique » du XIX ${ }^{\mathrm{e}}$ siècle et celle de l'historicisme. Nous devons certainement comprendre cette polémique à la lumière de son refus de se positionner pour ou contre l'une des deux conceptions prédominantes de la philosophie : d'une part, une tendance qui l'identifie à une théorie de la connaissance et, d'autre part, sa réduction à une " conception du monde ». La prétention de cette dernière à la " totalité » est un des thèmes-clés pour appréhender la critique sévère adressée aux concepts de «total» et de " forme ${ }^{50}$ ». Par conséquent, dans une démarche qui consiste à placer le jeune Heidegger au service du Heidegger ou « des Heidegger » qui vont suivre ${ }^{51}$, une continuité pourrait se dessiner entre sa critique de la postérité de Hegel et la

48. Ibid., p. 16.

49. Cf. J. Barash, Heidegger et son siècle, Paris, PUF, 1995, p. 28, 122.

50. GA 65, 14. Philosophie und Weltanschauung, p. 41. Le paradoxe repose ici sur le fait que la pensée spenglérienne de la crise et du déclin est le miroir inversé du projet heideggerien de Destruktion. À ce sujet : C.R. Bambach, Heidegger, Dilthey, and the Crisis of Historicism, Ithaca, Cornell University Press, 1995, p. 14, 16, 200; J. Barash, Martin Heidegger and the Problem of Historical Meaning, La Haye, Martinus Nijhoff, 1988, p. 150.

5I. " The young Heidegger - this Heidegger earlier than the early Heidegger — can be of service in finding the Heidegger or, better, the Heideggers later than the later Heidegger, as well as ways of thinking that no longer directly bear Heidegger's seal » (John van Buren, The Young Heidegger. Rumor of the Hidden King, Bloomington/Indianapolis, Indiana University Press, 1994, p. 26). 
confrontation ultérieure avec la philosophie nietzschéenne axée sur les motifs du système et de la négativité.

Certes, les nouvelles conditions qui régissent la confrontation heideggerienne avec la métaphysique après le tournant des années trente situe ce débat dans un registre sensiblement différent. Nous ne devons pas néanmoins négliger la continuité de sa démarche qui permet à un nombre de thématiques diverses de rester en suspens, de resurgir ou de se modifier, sans jamais s'effacer entièrement de l'horizon de sa pensée. Ce qui retiendra notre attention par la suite, c'est sa critique d'Oswald Spengler. Notre objectif sera de montrer que l'affinité sous-jacente établie entre Nietzsche et Hegel a comme arrière-plan l'appropriation de certains aspects de la philosophie nietzschéenne par les courants post-hégéliens de la philosophie de l'histoire. Ce rapprochement jettera une nouvelle lumière sur le rôle-clé du motif de la Gestalt dans l'interprétation du Surhomme, en montrant que son surgissement au moment culminant du " différend" (Auseinandersetzung) avec Nietzsche puise ses sources dans la première confrontation heideggerienne avec l'héritage hégélien dans ses cours et écrits de l'herméneutique de la facticité.

Nous nous proposons ainsi d'envisager cette première critique de Spengler comme le sol nourricier de son propos postérieur sur la Gestalt. En fait, le débat heideggerien avec le post-hégélianisme ne se confine point aux années de l'enseignement à Fribourg et puis à Marbourg mais revient à plusieurs reprises tout au long de son parcours ultérieur. Ainsi lit-on dans le cours de 1934/35 sur l'hymne hölderlien « Le Rhin » : « Aux confusions du XIX siècle appartient le fait de croire que la science de l'histoire [...] devrait rechercher [...] le général et ce qui peut se ramener à des lois. C'est tout à fait dans cette direction que pensent par exemple Spengler, et toutes les "morphologies", toutes les "typologies" de l'histoire ${ }^{22} "$.

Il s'agit donc pour Heidegger de se confronter avec la conception spenglérienne d'une morphologie des cultures censée être capable d'englober le devenir de l'humanité dans son ensemble. Cette critique a comme point de départ l'œuvre majeure de Spengler intitulée Le déclin de l'Occident. Esquisse d'une morphologie de l'histoire universelle (1917) qui s'articule en deux parties dont la première porte le titre suggestif pour notre propos « Forme et réalité » ( Gestalt und Wirklichkeit»). Elle s'effectue à plusieurs reprises depuis le tout début de l'enseignement à Fribourg en constituant un moment charnière dans la cristallisation de son projet herméneutique initial53. C'est le cas pour le dernier cours

52. Les Hymnes de Hölderlin: La Germanie et Le Rhin, Paris, Gallimard, 1988, p. 210.

53. GA 63, p. 35, 43-48. c'est dans le cours du semestre d'hiver 1921/22 intitulé Introduction à la Phénoménologie de la Religion qu'une première approche systématique de la philosophie spenglérienne de l'histoire apparaît (GA 60, p. 31, 41-54). Au sujet de cette « protoherméneutique » chez Heidegger : J.Grondin, « Die Hermeneutik der Faktizität als ontologische Destruktion und Ideologiekritik ", Zur philosophischen Aktualität Heidegger, II, Tübingen, 1990, p. 163-178; J. van Buren, "The Young Heidegger : Rumor of the Hidden King (19191926) ", Philosophy Today, 33 (1989). 
professé à Fribourg pendant le semestre d'été 1923 qui porte précisément le titre Ontologie (Herméneutique de la facticité) et qui, dans son troisième chapitre, traite de la philosophie spenglérienne de l'histoire.

Un des premiers propos qui portent sur ce sujet est celui du cours de 1920 intitulé Phänomenologie der Anschauung und des Ausdrucks. Theorie der philosophischen Begriffsbildung. L'analyse débute avec la désignation des deux démarches pensantes qui rendent possible l'interprétation du présent : d'une part, l'examen philosophique et, d'autre part, l'étude de la conscience historique du présent. À la place du terme de morphologie, Heidegger utilise parfois les expressions de "Wie des Ausdruckseins » ou de " jeweilige Stil der Kultur ». Nous devons aussi faire allusion sur ce point à une autre critique importante, adressée cette fois-ci contre la typologie jasperienne des « conceptions du monde » (Weltanschauungen), puisque le terme de Gestalt apparaît aussi à cette occasion. C'est dans le cadre de cette critique que resurgit l'opposition entre le «systématique » et le facticiel, la typologie et l'existence singulière ${ }^{54}$.

La philosophie de la culture correspond à cette démarche de systématisation. Le devenir-de-la-forme (Gestaltwerdung) ainsi que l'êtreexprimé (Ausdrucksein) d'une culture constituent selon Spengler des modes de présentification du passé à travers l'objectivation de l'élément subjectif qui est l' «âme d'une culture » (Kulturseele) $)^{55}$. D'après Heidegger, les deux points de départ de toute considération sur la vie sont, d'une part, la vie comme acte d'objectivation, de formation de quelque chose (etwas Gestalten), ce qui correspond à la quête de la Kulturphilosophie et, d'autre part, la vie comme le fait d'éprouver un vécu (Erleben). Ce deuxième versant fait certes allusion à la philosophie de la vie et du vécu dont W. Dilthey et G. Simmel étaient les représentants principaux.

Bien que la possibilité d'une recherche scientifique de la conscience historique n'y soit pas exclue, la qualité essentiellement formatrice (gestalthafte) du passé objectivement saisissable est explicitement niée par Heidegger. La raison

54. Heidegger remarque à propos de la cohésion de la vie (Lebenszusammenhang) chez Dilthey : "Dilthey sieht das Seelische [...] als eine Gestalt, zuständlich, "ästhetisch" [...] " (Phänomenologie der Anschaunng und des Ausdrucks. Theorie der philosophischen Begriffsbildung, 1920, GA 59, Francfort, Klostermann, 1993, p. 28); cf. « Remarques sur la "Psychologie des Weltanschaunngen" de Karl Jaspers ", Philosophie, n 12, 1986. À ce sujet : R.A. Makkreel, "Heideggers ursprüngliche Auslegung der Faktizität des Lebens : Diahermeneutik als Aufbau und Abbau der geschichlichen Welt » in : Zur philosophischen Aktualität Heidegger, p. 183-184.

55. Ibid., p. 39. Le "devenir-de-la-forme " (Gestaltwerdung) se distingue nettement d'une édification de l'avenir "dans la nouvelle création du Dasein lui-même " (in einer Neuschaffung des eigenen Daseins), propos dont le ton nietzschéen est manifeste (GA 60, p. 54). Ce projet trouvera sa formulation complète dans les derniers cours professés à Fribourg : "Philosophie als prinzipielle Erkennen ist nichts anderes als der radikale Vollzug des Historischen der Faktizität des Lebens, so dass in ihm und für es Geschichte und Systematik gleich fremd und in ihrer Scheidung ebenso und erst recht überflüssig sind »(GA 61, p.111). Si la «typification " (Typisierung) témoigne de l'esthétisation de l'expérience historique comme pure inversion du primat de la connaissance objective qui ne fait à la fin que l'affirmer (ibid.), la «nouvelle création" du Dasein nous renvoie à l'appropriation originaire facticielle de l'historique (die ursprüngliche faktische Aneignung des Historischen) (p. 110). 
en est que celle-ci se rapproche plutôt de la philosophie des valeurs qualifiée de platonisme des barbares ${ }^{56}$. La Gestalt constitue pour Spengler le caractère objectif du passé qui impose un ordre universel des cultures, dénoncé dans le cours de 1923 sur Ontologie (Hermeneutique de la Facticité) comme conscience historique inauthentique. Les modes divers de l'être-exprimé (Ausdrucksein) sont désignés comme étapes dans le "processus de la systématique elle-même " (Stationmarkierung im Prozess der Systematik selbst) ${ }^{57}$. La démarche spenglérienne consiste en effet à évoquer d'abord le style (Stil) de chaque culture et, par la suite, à mettre en rapport les cultures entre elles dans leur essence formatrice (gestalthaft) afin qu'on puisse parvenir à l'universalité d'une réflexion sur le phénomène de l'histoire (die Universalität der Geschichtsbetrachtung) ${ }^{58}$. Cette compréhension des cultures se qualifierait aussi de biologique : celles-ci représenteraient en effet des plantes, des organismes, d'où la possibilité d'un parallélisme entre leur genèse et et l'évolution organique. Dans son entreprise définie aussi comme l'idée d'une "symbolique universelle ", Spengler a comme prédécesseurs Nietzsche, Bergson, Dilthey et Lamprecht. Spengler figure ainsi comme un penseur de l'histoire : sa réflexion est " philosophie de l'histoire sans l'historique »(Geschichtsphilosophie obne das Historische). Le point critique pour notre examen de la Gestalt est certainement le renvoi de la pensée de l'histoire chez Spengler à son arrière-plan hégélien ${ }^{59}$. Au processus dialectique, Heidegger oppose ce qu'il nomme dans le cours du semestre d'hiver 1919/20 une "diaherméneutique ${ }^{60}$ » mettant en avant le caractère facticiel de la vie, l'ouverture d'une compréhension de soi concrète en rapport avec le Dasein de la vie, interrogation qui inaugure sous plusieurs aspects le projet de l'ontologie fondamentale ${ }^{61}$.

56. GA 63, p. 42. À travers le "devenir-de-la-forme ", c'est le modèle platonicien de la mimésis qui prévaut (GA 60, p. 45), même dans le cas où le contraire est affirmé : " Alle frei Wege sind grundsätzlich durch die Platonische Auffassung durchherrscht, auch Spengler, der nur im Gegensatz zu ihm die geschichtliche Wirklichkeit verabsolutiert " (p. 47; souligné dans le texte).

57. Ibid., p. 61. Heidegger explicite cette idée de multiples façons. Il parle d' "Ausdruck eines etwas von etwas ", de " jeweilige Gestaltart des Ausdruckseins ", de "Vollziehbarkeit eines universalen gestaltgleichenden Ordnens ".

58. GA 59, p. 28.

59. Grundprobleme der Phänomenologie, GA 58, Francfort, Klostermann, 1992, p. 263. Il est important de remarquer que d'emblée le projet d'une destruction de l'ontologie dont la « diaherméneutique » est la première figure se situe aux antipodes de la dialectique; cf. Ontologie (Hermeneutik der Faktizität), 1923, GA 63, p. 150-151.

60. Ceci est d'autant plus stimulant que la " diaherméneutique " s'exprime aussi parfois dans les termes d'une figuration créatrice (Gestaltung) du monde de la vie. Nous signalons qu'aussi tôt que dans le cours de 1919/20, Heidegger parle de la figurativité expressive de la vie déterminant le mode de l'expérience en incorporant le facticiel et le singulier qui n'est certes pas à confondre avec l'universalité de la "forme " imprégnée de négativité dialectique chez Spengler (GA 58, p. 45, 137, 139 etc). Cf. J. Greisch, "La "tapisserie de la vie”. Le phénomène de la vie et ses interprétations dans les Grundprobleme der Phänomenologie de Martin Heidegger ", Heidegger 1919-1929. De l'herméneutique de la facticité à la métaphysique du Dasein, Paris, Vrin, 1996, p. 140, 142.

6I. GA 63, p. 38. 
Cependant, ici comme ailleurs et même dans les textes rédigés peu après 1927, Nietzsche n'est en aucun cas rangé parmi les philosophes de la culture et de la $v^{2} e^{62}$. L'axe de ces courants était l'élaboration, d'une part, d'une "systématique » de l'histoire et, d'autre part, d'une conception biologique qui fait de l'histoire un processus organique. Ce qui demeure étonnant, c'est que nous retrouverons cette même duplicité par la suite dans l'unité inextricable des deux traits du Surhomme, sa désignation, d'une part, comme Gestalt et, d'autre part, comme Esprit inversé en animalité de la Volonté de puissance. Si la critique de la philosophie post-hégélienne dans les cours du début des années vingt n'établit aucun rapport avec Nietzsche, ceci ne sera plus le cas par la suite. En effet, dans les cours des années quarante, le rapprochement de Nietzsche et de Spengler est révélateur d'un tournant dans le " différend ${ }^{63}$ ». Il devient apparent que, désormais, l'interprétation de Nietzsche se formule dans des termes proches de la critique adressée par Heidegger à la philosophie post-hégélienne de l'histoire.

Ainsi, dans le cours de 1942/43 sur Parménide, il distingue nettement entre l'histoire (Geschichte), déterminée à partir de l'essence de la vérité, et l'enquête ou la science historique (Historie) susceptible d'un processus d'objectivation. Nietzsche se situe du côté de la quête historique qui mine l'authenticité de l'histoire (Geschichte): son projet d'inversion des valeurs correspond à la forme d'objectivation propre au calcul (Verrechnen). Nietzsche s'enquiert en effet de la possibilité de donner un sens à l'histoire au même titre que les courants post-hégéliens de la philosophie de l'histoire. Or, il n'est guère fortuit que cette démarche de donation de sens (Sinngebung) à l'histoire, qui caractérise la philosophie de l'histoire de la fin du XIX ${ }^{\mathrm{e}}$ siècle dont Spengler est une des figures principales, constituera par la suite un des nœuds de la critique heideggerienne du Surhumain ${ }^{64}$.

Pour conclure ces réflexions, nous tenons à revenir sur ce que nous avons déterminé comme la fonction singulière du motif de la Gestalt dans l'interprétation heideggerienne de Nietzsche. Ses origines et ses enjeux sont à chercher en avant dans les premières années de son enseignement et ils se prolongent jusqu'à la fin. Dans la mesure où sa critique de la conception morphologique de l'histoire chez Spengler s'est associée dès le tout début avec sa propre interrogation sur les conditions ontologiques de la compréhension historique, le motif de la Gestalt imprègne inéluctablement sa détermination de l'historicité. Le terme d'empreinte (Prägung) désigne ici l'époqualité de la métaphysique, soumise à l'impératif de la totalité. Ce qui est pourtant étonnant, c'est que ce même faisceau sémantique de l'empreinte et de la forme est utilisé

62. On pourrait parler à cet égard d'un premier geste appropriant envers Nietzsche. Ce sera le cas notamment pour Être et Temps ( tr. E. Martineau, 1985, Paris, Authentica, p. 271272). À ce sujet : J. Taminiaux, «La présence de Nietzsche dans Sein und Zeit » dans Lectures de l'ontologie fondamentale, Grenoble, J. Millon, 1995.

63. Cf. "Die einzigen, der Vollendung der Neuzeit zustrebenden und der Beachtung würdigen Ausfaltungen der letzten abendländischen Metaphysik — derjenigen Nietzsches sind O. Spenglers Geschichtsmetaphysik des Caesarismus und E. Jüngers Metaphysik des "Arbeiters" [...] »(Besinnung, GA66, p. 27; souligné dans le texte). Aussi : GA 54, p. 101. 64. Ibid., p. 83. 
pour illustrer sa propre réflexion sur les époques de l'histoire de l'Être. Ainsi, dans la conférence de 1956/57 sur La constitution onto-théo-logique de la métaphysique, il sera question de forger des " figures dispensionnelles de l'Être " qui échappent à la généralité des représentations historiques en raison de leur qualité de singulare tantum:

Il n'y a jamais d'être si ce n'est chaque fois marqué de telle empreinte à lui

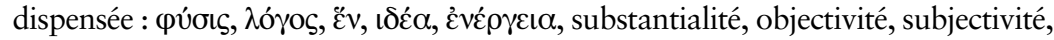
volonté, volonté de puissance, volonté de volontée ${ }^{65}$.

$\mathrm{Vu}$ sous un autre angle, le registre de la Gestalt appartient au lexique de l'Ereignis ${ }^{66}$. Il importe donc de signaler l'état critique de l'ambivalence qui marque le statut de la Gestalt. On y voit en effet opérer, d'une part, la confrontation de Heidegger avec la métaphysique et, d'autre part, la mise en place de sa propre réflexion sur l'Être. Dans cette tension, c'est l'ombre de Nietzsche qui surgit constamment à l'arrière-plan.

65. Questions I, Paris, 1968, p. 301; cf. ibid., p. 270, 300.

66. "Le simple de l'estre (Seyn) a en soi l'empreinte (Gepräge) de l'unicité. Elle n'a nullement besoin de distinction et de différences, même pas de la différence avec l'étant " (GA 65, p. 471; trad. J. Greisch dans Rue Descartes : "Des Grecs », 1, 1991, p. 215). 